\title{
CLASES MEDIAS Y TRABAJADORES FRENTE A LA POLÍTICA. SOBRE EL ASCENSO ELECTORAL DE LA DERECHA EN CHILE
}

\author{
Middle classes and workers againt the policy. \\ About the rise of right-wing political in Chile \\ Mayarí Castillo Gallardo*
}

\begin{abstract}
RESUMEN
El artículo tiene por objetivo comprender el ascenso de la derecha en Chile en la última década, a partir del análisis de dos segmentos: las clases medias y los trabajadores en la base de la estratificación social de la ciudad de Santiago. A más de tres décadas de la aplicación de las políticas de ajuste estructural, ambos sectores experimentaron transformaciones que impactaron de en los procesos de articulación de identidades políticas. La hipótesis que se sustenta en este artículo es que estos cambios en la estratificación social han delimitado el surgimiento de nuevas formas de articulación identitaria, un terreno fértil para organizaciones políticas cuyo accionar se orienta hacia una intervención en lo local, una dinámica clientelar y por liderazgos personalistas, elementos fundamentales en la estrategia política de los partidos de derecha en Chile. Para sustentar la hipótesis, se expone en una primera etapa un análisis cuantitativo en el cual se cruzan datos de la Encuesta de Caracterización Socioeconómica Nacional (CASEN) 2006 con datos electorales de las elecciones del 2005 y 2006. En una segunda etapa, se expone un análisis cualitativo basado en la descripción de la trayectoria y elementos principales de las articulaciones identitarias de los dos segmentos elegidos, estableciendo su impacto para el fenómeno político.
\end{abstract}

Palabras clave: Voto, identidades, clases medias, trabajadores, partidos de derecha, Chile.

\footnotetext{
* Escuela de Antropología, Universidad Academia de Humanismo Cristiano / Centro Interdisciplinario de Estudios Interculturales e Indígenas (ICIIS). Santiago, Chile. Correo electrónico: mcastillog@docentes.academia.cl

Artículo recibido el 19 de diciembre de 2012. Aceptado el 9 de abril de 2014 .
} 


\begin{abstract}
This paper aims understanding the rise of right-wing political sectors in Chile during the last decade through the analysis of two social groups: the middle classes and workers in Santiago City. More than three decades after the liberal structural reforms, both sectors have experienced crucial transformations, which heavily impacted in their political identification processes. The proposed hypothesis is that these changes in social stratification - dependent on the reforms- established new forms of identity articulation which are particularly compatible with political organizations with certain features: emphasis in the local space, clientelistic dynamics and leaderships based in the idea of the "apolitical", all these core elements of the political proposal of right-wing parties in Chile. To sustain this hypothesis, set out in a first step a quantitative analysis with CASEN 2006 data and the election data from 2005 and 2006 presidential elections. In a second step, this paper presents a qualitative analysis based on the description of historical trajectories and the main elements of the articulations of the identity the selected groups, establishing their impact on the political phenomenon.
\end{abstract}

Keywords: Vote, identities, middle classes, workers, right-wing parties, Chile.

\title{
INTRODUCCIÓN
}

La victoria del candidato presidencial de derecha en Chile en el año 2009 despertó sorpresa a nivel nacional y latinoamericano. No sólo mostró una tendencia opuesta a la observada en el resto de los países del cono sur, sino que también implicó una excepción en la historia nacional: sólo por segunda vez en toda la vida republicana y por primera vez después de finalizada la dictadura militar, un candidato de derecha era electo democráticamente para desempeńar el cargo de presidente.

La sorpresa era justificada y los análisis destinados a explicar la situación numerosos. Sin embargo, pocos de ellos apostaron por una mirada de largo plazo, que iluminara las tendencias de las últimas décadas en las que el aumento del apoyo electoral de la derecha ha sido una tendencia que se ha manifestado con mayor fuerza aún en las elecciones municipales y parlamentarias (Morales y Cantillana, 2008; Rojas, 2008; Altman, 2004). Ya sobre las elecciones presidenciales del año 2000, la investigación había problematizado el aumento del voto de derecha en determinados sectores sociales, relacionándolo con las transformaciones de este sector político y con un reordenamiento de los clivajes que habían ordenado el escenario político chileno a partir del período de transición (Tironi et al. 2001; Torcal y Mainwaring, 2003; Altman, 2004). En estos análisis, la evidencia apuntaba a una mayor penetración de la coalición de derecha en el sector medio y bajo, mientras que la coalición de centro izquierda perdía importante terreno en los sectores populares (Tironi et al., 2001:77; Morales y Cantillana, 2008: 2-3; Rojas, 2008; Altman, 2004; Aninat y Elacqua, 2010).

Ambos cambios iban en una línea directamente contraria a las tendencias estudiadas en el sistema político chileno, caracterizado por definirse en torno a los ejes clase y religión, sumado al nuevo clivaje del periodo post dictatorial que dividía el espectro

político en torno al binomio democracia/autoritarismo (Scully, 1992; Mainwaring y Torcal, 2003; Valenzuela, 1995; Valenzuela, 1996). Relativizando estas tendencias y 
matizando su vínculo con la dictadura militar, el partido Unión Demócrata Independiente logró convertirse en el partido más votado en las elecciones parlamentarias del 2001-2005 y la coalición de derecha llegó a disputar el sillón presidencial en segunda vuelta, siendo derrotada por sólo 2,6\% del total de los votos válidamente emitidos (Tironi et al, 2001). Este fenómeno se repitió en las elecciones del año 2005 y tuvo su punto culmine en las elecciones del 2009, con el triunfo de Sebastián Pińera.

En ese marco, hay tres factores asociados al aumento del apoyo de la coalición de derecha en Chile en las últimas elecciones y cada uno de ellos representa una vía para acercarse al problema. El primero de ellos apunta al proceso de desgaste de la coalición gobernante, en particular en la pérdida de apoyo en los sectores medios y populares. El segundo de ellos refiere a la transformación de la derecha, entendiendo ésta como un sector político complejo, compuesto por dos partidos con tradiciones ideológicas disímiles y trayectorias diferenciadas. El tercero se relaciona con un proceso de transformación de determinados sectores de la sociedad chilena y de su forma de relacionarse con la política, que pareciera ir modelando nuevas formas de articulación en los clivajes ya existentes y marcando el surgimiento de otros nuevos. Respecto al primer factor existe investigación al respecto. La mayor parte de los análisis electorales de la última década se han enfocado a documentar las transformaciones observadas en el apoyo de la coalición de centro izquierda, así como al análisis de los cambios a nivel diferenciado del voto de cada partido por grupo socioeconómicos (Aninat y Elacqua, 2010).

El segundo factor -la transformación de la derecha- también ha recibido algo de atención (Morales y Cantillana, 2008; Joignant y Navia, 2003; Huneuus, 2001; Soto, 2001; Arriagada, 2005; Castillo, 2009; Rojas, 2008; Cristi, 2008). En este campo se ha enfatizado la necesidad de diferenciar entre los partidos que componen el bloque de derecha, relevando los elementos que establecen diferencias claves en términos de trayectorias y plataforma política (Correa, 2004; Morales y Cantillana, 2008; Rojas, 2008). Dentro de estas diferencias y en relación al aumento del apoyo electoral del bloque, se ha investigado particularmente el aumento de la inserción del partido Unión Demócrata Independiente en los sectores populares (Morales y Cantillana, 2008; Tironi et al; 2001; Cristi, 2008).

Es en torno al tercer factor que hasta ahora no existe investigación que permita cerrar el puzzle del triunfo de la derecha en las últimas elecciones. Para cerrar este puzzle, es preciso comprender lo que subyace tras el comportamiento electoral de los individuos: transformación de largo aliento que implicaron un aumento del apoyo electoral a la derecha en sectores tradicionalmente vinculados a una tendencia de centro o de izquierda. Para que los dos primeros factores mencionados se tradujeran en un incremento de la derecha en las urnas, es necesaria una cierta receptividad de sectores sociales que tradicionalmente habían concebido su participación política y su relación con los partidos desde otro lugar. Los datos electorales indican que parte importante de los votos que se movieron hacia la derecha en las últimas elecciones fueron los de aquellos segmentos históricamente ligados a los partidos de centro o de izquierda: las clases medias y los trabajadores en la base de la estratificación social. En esta línea, el estudio de Aninat y Elacqua (2010) marca una línea sugerente, aunque incompleta, al identificar en la clase media "emergente" el electorado que decidió las elecciones del 2009. 
Partiendo de este dato, el artículo busca explorar qué hay detrás del cambio en el comportamiento electoral en estos sectores, a partir de la hipótesis de que éstos han desarrollado nuevas formas de articulación identitaria a partir de las modificaciones observadas en términos estructurales a partir de los ochenta. Estas transformaciones han ido delimitando nuevas formas de relacionarse con el espacio político, particularmente compatibles con aquellas organizaciones marcadas por un discurso que desplaza la centralidad de la clase, relevan la importancia de la movilidad social y establecen la dimensión local como espacio primordial de intervención, fomentando una mayor receptividad a liderazgos locales y desencajando la relación histórica de estos sectores con determinadas organizaciones políticas. Se ha ido estableciendo así un terreno fértil para la penetración de nuevas formas de accionar en lo político y nuevas interpelaciones discursivas, como las que han venido estructurando Renovación Nacional y la Unión Demócrata Independiente, desde su surgimiento en los años ochenta (Soto, 2001; Joignant y Navia, 2003; Huneeus, 2001; Arriagada, 2005; Castillo, 2009; Cristi, 2008; Dávila y Fuentes, 2002).

La elección de los segmentos para un análisis -clases medias y clase trabajadoraobedece a varias razones. En primer lugar, a que ambos segmentos han sido apuntados como los que han mostrado un mayor incremento en el apoyo electoral de la derecha en las últimas elecciones (Morales y Cantillana, 2008; Cristi, 2008; Altman, 2004: Aninat y Elacqua). En segundo lugar, tanto las clases medias como los trabajadores en la base de la estratificación social han sido seńalados en la literatura especializada como históricamente ligados al centro y a la izquierda para el caso chileno, en el marco de un sistema político en el cual el clivaje de clase es uno de los más relevantes (Valenzuela, 1995; Valenzuela, 1996; Faletto, 1981: Lomnitz y Melnick, 1998; Faletto, 1980). Finalmente, es en éstos segmentos en los que se puede ver con una mayor claridad las modificaciones operadas en la sociedad chilena a partir de la década de los ochenta ya que son quiénes fueron impactados con mayor fuerza por las políticas de ajuste, provocándoles cambios que posteriormente se consolidaron a partir del período de transición (Martínez y Tironi, 1985; León y Martínez, 2001; Atria, 2004; Barozet y Espinoza, 2008: Portes y Hoffmann, Sémbler, 2006; Silva, 2005; Winn, 2004) ${ }^{1}$.

Para realizar este análisis, se han utilizado dos tipos de datos: cuantitativos y cualitativos. Los primeros se usaron para documentar a modo contextual qué segmentos de la sociedad chilena habían mostrado un aumento del voto de derecha en la última elección en relación a la elección del 2005. Se utilizaron datos a partir de la encuesta CASEN del año 2006, los que se correlacionaron con los resultados oficiales de las elecciones del año 2005 y 2009, sobre votos válidamente emitidos.

\footnotetext{
${ }^{1}$ Estas modificaciones se pueden observar principalmente en tres aspectos: 1) Cambios en sector económico: En el caso de las clases medias, se constata un importante tránsito del sector público al privado, marcado por la contracción del gasto público, así como un aumento de la población de pequeńos propietarios (Barozet y Espinoza, 2008; Sémbler, 2006; Silva, 2005). En el segundo caso, hay un tránsito desde el sector primario al sector servicios, generado por la pérdida de importancia del sector industrial a nivel nacional y regional (Martínez y Tironi, 1985; León y Martínez, 2001, Winn, 2004) 2) Tipo de relación laboral y empleo. En ambos segmentos es posible observar modificaciones marcadas por una tendencia a la flexibilización y precarización laboral, siendo esta última instalada como fenómeno general por la modificación de los códigos laborales en el marco del paquete de reformas estructurales (Sisto y Fardella, 2009; Minujin y Comas, 2009; Barozet, 2006) 3) Movilidad social. Las tendencias para los grupos observador son a una movilidad ascendente y horizontal, delimitada sobre todo por el aumento de la escolaridad y el cambio en la matriz económica a partir de los ochenta (Espinoza, 2006; Torche, 2005; Torche y Worlmald, 2007; Espinoza et al, 2012; Méndez y Gallo, 2007). En ese marco, los patrones de movilidad observados apuntan a un aumento del flujo entre posiciones, sobre todo si se analiza el fenómeno en términos intergeneracionales y en el caso de las clases medias (Méndez, 2006; Barozet y Fierro, 2011).
} 
El enfoque con el cual se trabajó los datos de la CASEN fue el análisis de clases basado en el trabajo de E. O. Wright $(1985 ; 2009)^{2}$, a partir de cuyo esquema y en base a los grandes grupos del código CIUO se establecieron los siguientes segmentos de clase: 1) Clase alta: Miembros del poder ejecutivo y de los cuerpos legislativos, personal directivo de la administración pública y de empresas 2) Clase media clásica: profesionales, científicos e intelectuales, técnicos y profesionales de nivel medio) 3) Clase media baja: Empleados de oficina, Trabajadores de los servicios y vendedores de comercios 4) Clase trabajadora: Agricultores y trabajadores calificados agropecuarios y pesqueros; oficiales, operarios y artesanos de artes mecánicas y de otros oficios con calificación calificados, operadores de instalaciones y máquinas y montadores (calificados/ semi calificados), trabajadores no calificados. A partir de estos datos, se clasificaron las comunas del Gran Santiago de acuerdo a la proporción de jefes de hogar económicamente activos que pertenecían a las distintas categorías de clase, considerando el peso que estas clases poseían en el total de la población. Estos se correlacionaron con los datos electorales oficiales del Ministerio del Interior del Gobierno de Chile, para las elecciones del 2005 y 2009.

Los datos cualitativos se basan el análisis de 24 entrevistas en profundidad, unas de elaboración propia y otras puestas a disposición pública de la comunidad científica por el Centro de Investigación en Estructura Social de la Universidad de Chile (CIES), ambos tipos de entrevistas realizadas en el año $2008^{3}$. Son entrevistas de 8 mujeres y 16 hombres, segmentados por clases a partir del modelo antes expuesto. Dentro de éstos, hay 14 personas clasificadas como clase media clásica, 2 de clase media baja y 8 de clase baja, entre 28 y 64 años. La información mediante el software Nvivo y a través de la metodología de la Teoría Fundamentada de Glaser y Strauss (1998), que contempla una primera etapa de codificación libre y luego la generación de modelos teóricos de mediano alcance a partir del refinamiento paulatino de códigos. Este proceso dio por resultado dos categorías de mediano alcance: Autoidentificación en el espacio social - Socialización y Acción política, con las cuales se trabajó para este artículo ${ }^{4}$.

\footnotetext{
${ }^{2}$ Inscribiéndose en una tradición teórica neomarxista, el trabajo de Wright permite visibilizar también elementos propios de un análisis de carácter más weberiano relevantes como son los niveles de control sobre el proceso laboral y niveles de cualificación, permitiendo un análisis mucho más complejo que el que podría producir un análisis como los producidos con una segmentación por Grupos Socioeconómicos (GSE) o ingreso, basada principalmente en la capacidad y patrones de consumo. Desde este tipo de categorizaciones, las diferencias entre clase media y clase alta se reducen al ingreso, lo que invisibiliza el rol del patrimonio, la cualificación, el nivel de control sobre el proceso productivo, que establece diferencias claves entre sujetos. Así, en las clasificaciones por ingreso o GSE un profesional de alto ingreso se encuentra en la misma categoría que quiénes poseen un ingreso similar, pero que tienen participación en las empresas en las que trabajan y se encuentran en una posición superior en términos de control y supervisión, aún cuando la posición de ambos es visiblemente diferente. Este problema fundamental de las clasificaciones por ingreso y consumo son las que han dirigido la investigación a trabajar con el modelo de Wright.

${ }^{3}$ Las entrevistas aplicadas por la investigadora eran semiestructuradas y estaban dirigidas principalmente a tres aspectos: identificación y autopercepción/ definición y percepciones sobre clases/ identificación y acción política. Las entrevistas puestas a disposición del CIES eran de una duración aproximada de una hora y estaban enfocadas principalmente a identificación y autopercepción/ definición y percepciones sobre desigualdad.

${ }^{4}$ La primera de ellas está compuesto de tres dimensiones: el yo en el espacio social/ el otro "opuesto" en el espacio social/ el similar o "cercano" en el espacio social. En análisis se buscó los elementos articuladores de cada dimensión en el discurso de los entrevistados y su relación con las transformaciones observadas en términos estructurales. La segunda categoría se compuso tres dimensiones: Socialización política inicial/ socialización política secundaria/ acción política. En las dos primeras se puso especial atención en documentar los espacios y sujetos relevantes en la conformación de una opinión y posición política de los sujetos, con el fin de identificar aquellos cambios que podrían estar en la base del cambio del comportamiento electoral de estos sectores. En la tercera dimensión se agruparon los elementos del discurso del sujeto referidos al tema de la acción política, entendiendo ésta como cualquier acción realizada de manera consciente con el fin de influir en el sistema político. En esta dimensión se consideró relevante incluir espacialmente tres fenómenos: percepciones de acción política legítima, percepciones sobre la propia acción política y percepciones sobre el alcance e influencia de la acción política.
} 


\section{LA DERECHA Y EL VOTO DE CLASE: ANÁLISIS DEL GRAN SANTIAGO}

Existe un cierto consenso en la literatura en la importancia que uno de los clivajes más relevantes del sistema político chileno durante buena parte del siglo XX fue la clase o la posición socioeconómica de los sujetos. Si bien con algunos matices, la literatura al respecto tiende a coincidir con que la variable socioeconómica fue uno de los ejes, sino quizás el principal, que articuló la política en Chile durante el siglo XX (Zeitlin y Petras, 1970; Faletto, 1982; Scully, 1992; Valenzuela, 1995, Torcal y Mainwaring, 2003).

Respecto al vínculo entre partidos de derecha y élites existe también un consenso establecido, aún cuando las diferencias internas de las élites chilenas hayan dado lugar a tradiciones políticas diferenciadas (Correa, 2004; Fediakova, 2002; Morales, 2004; Moulian y Torres, 1989). En el caso de la izquierda y el centro, a pesar de que la fortaleza de la relación ha sido considerada menos intensa, se ha estableció un vínculo histórico entre éstos y los trabajadores y las clases medias, respectivamente (Johnson, 1971; Zeitlin y Petras, 1970; Scully, 1992; Faletto, 1980). Así, si bien es posible discutir con estas asociaciones tan unívocas respecto al componente estructural y representación política (Castillo, 2011), se puede decir que la tendencia general documentada en la investigación pertinente remite a un notorio vínculo histórico entre voto de izquierda/centro y trabajadores/clases medias, respectivamente. Para el período posdictatorial, por otro lado, se ha afirmado la continuidad de este patrón en la configuración del sistema político chileno (Valenzuela, 1995), señalando que las primeras elecciones arrojan datos contundentes en torno a la mantención de la relación histórica entre los partidos y sus bases de apoyo en términos de la variable socioeconómica 5 .

En ese sentido y considerando que la literatura al respecto muestra lo arraigado que se encuentra esta relación entre comportamiento electoral/ posición de clase en el sistema político chileno, el triunfo de la derecha parece aún más enigmático. Para explicarlo es preciso atender a los signos o indicadores de transformaciones de más largo plazo, tales como la rearticulación de la relación entre el partido de derecha Unión Demócrata Independiente y los sectores populares, basada en una forma de clientelismo incipiente que ha proliferado a partir de una transformación de la manera en que estos sujetos se relacionan con la política, como han apuntado algunos estudios de caso al respecto (Soto, 2001; Arriagada, 2005; Castillo, 2009). Atendiendo tanto a la mantención del peso de la variable socioeconómica se hace preciso investigar de qué manera los cambios recientes en términos de estratificación social están influyendo en estos cambios. Este trabajo pretende ser un aporte en esta dirección ${ }^{6}$.

\footnotetext{
${ }^{5}$ Esto no significa que no se hayan registrado cambios significativos al respecto en la última década. El estudio de Torcal y Mainwaring (2003) ha mostrado cómo la relación histórica entre clases y apoyo electoral ha ido declinando a partir del período de transición, apuntando a que la importancia de la variable socioeconómica es menos visible para el caso determinados partidos políticos, aún cuando sigue siendo estructurante para el binomio Coalición de derecha/ Coalición de centro - izquierda. La evidencia aportada por Tironi et al. (2001) apunta también en esta dirección.

${ }^{6}$ Cabe señalar que para efectos de este artículo no se establecieron diferencias en el aumento del apoyo electoral de uno u otro partido de derecha, ya que se trabajó con los resultados de la segunda vuelta electoral, los que se encuentran agregados por coalición y no por partido. Esta dificultad metodológica y la necesidad de distinguir dentro de la base de apoyo de la derecha actual, hace preciso en un futuro avanzar hacia análisis basados en el comportamiento electoral diferenciados por partidos, lo que puede ser llevado a cabo a partir de las elecciones parlamentarias o municipales.
} 
En esta línea, los resultados cuantitativos obtenidos muestran que en las comunas del Gran Santiago el porcentaje de votos obtenidos por la derecha en las elecciones presidenciales del 2005 se siguen relacionando significativamente con su composición de clase. La mayor presencia de jefes de hogar de clase media baja o baja tuvo un impacto negativo en el voto de la coalición de derecha, mientras que la mayor presencia de jefes de hogar de clase media clásica o alta fortaleció al sector. Esta relación se mantiene en las elecciones del 2009. Sin embargo, es preciso señalar que todas estas relaciones disminuyeron en esta última elección: el coeficiente de correlación de Pearson entre el voto de derecha y la presencia de la clase baja era de -.6851 en el 2005 y de -.6672 en el 2009. Entre el voto de derecha y la clase media-baja era de -.6199 en 2005 y -.6107. Entre el voto de derecha y la clase media clásica los coeficientes obtenidos pasaron de .7323 a .7126 en el mismo período. En esta marco, es posible decir que si bien esta asociación negativa entre derecha y clase baja/ media baja siguió siendo significativa, se difuminó en las últimas elecciones. Lo mismo para el caso de la asociación entre clase media clásica y apoyo a los partidos de derecha. Este fenómeno es uno de los indicadores de modificaciones que, si bien aún de manera incipiente, comienzan a manifestarse.

Si se analizan los datos desagregados para el Gran Santiago es posible observar que las comunas que muestran un aumento sobre el $6 \%$ en el apoyo electoral de la derecha, son comunas en las que la cantidad de hogares clasificados como clase baja va entre un $55.9 \%$ aun $71.4 \%$ como Puente alto, Huechuraba, Quilicura, Peńalolén, Pudahuel o Cerro Navia. 


\section{Cuadro 1. Comunas por porcentaje que jefes de hogar clasificados como clase baja y votación de derecha.}

\begin{tabular}{|c|c|c|c|}
\hline Comunas & $\begin{array}{c}\% \text { Jefes de hogar de } \\
\text { clase baja }\end{array}$ & Derecha 2005 & Derecha 2009 \\
\hline Padre Hurtado & $74,4 \%$ & $40,46 \%$ & $45,20 \%$ \\
\hline Pirque & $73,8 \%$ & $57,03 \%$ & $60,19 \%$ \\
\hline La Pintana & $72,7 \%$ & $36,50 \%$ & $41,57 \%$ \\
\hline Cerro Navia & $71,4 \%$ & $37,39 \%$ & $43,80 \%$ \\
\hline La Granja & $71,2 \%$ & $39,30 \%$ & $44,33 \%$ \\
\hline San Ramón & $69,1 \%$ & $39,47 \%$ & $44,01 \%$ \\
\hline San Bernardo & $69,0 \%$ & $44,69 \%$ & $50,66 \%$ \\
\hline San José De Maipo & $68,9 \%$ & $45,21 \%$ & $51,46 \%$ \\
\hline Lo Prado & $67,4 \%$ & $39,53 \%$ & $45,41 \%$ \\
\hline Renca & $66,2 \%$ & $39,45 \%$ & $45,47 \%$ \\
\hline El Bosque & $64,1 \%$ & $43,95 \%$ & $49,62 \%$ \\
\hline Huechuraba & $63,3 \%$ & $39,13 \%$ & $47,06 \%$ \\
\hline Peñalolén & $62,0 \%$ & $40,25 \%$ & $47,11 \%$ \\
\hline Lo Espejo & $61,3 \%$ & $37,42 \%$ & $40,98 \%$ \\
\hline Cerrillos & $61,1 \%$ & $47,76 \%$ & $50,96 \%$ \\
\hline Recoleta & $60,9 \%$ & $43,49 \%$ & $49,36 \%$ \\
\hline Conchalí & $58,6 \%$ & $42,18 \%$ & $48,11 \%$ \\
\hline Quilicura & $58,2 \%$ & $41,47 \%$ & $48,81 \%$ \\
\hline Quinta Normal & $57,5 \%$ & $42,68 \%$ & $48,40 \%$ \\
\hline Pudahuel & $57,3 \%$ & $39,09 \%$ & $45,56 \%$ \\
\hline Pedro Aguirre Cerda & $56,9 \%$ & $36,53 \%$ & $40,37 \%$ \\
\hline Estación Central & $56,6 \%$ & $41,84 \%$ & $47,00 \%$ \\
\hline Puente Alto & $55,9 \%$ & $42,26 \%$ & $48,80 \%$ \\
\hline San Joaquín & $52,2 \%$ & $38,81 \%$ & $43,63 \%$ \\
\hline Independencia & $49,2 \%$ & $46,63 \%$ & $52,86 \%$ \\
\hline Macul & $49,0 \%$ & $42,82 \%$ & $48,56 \%$ \\
\hline Lo Barnechea & $49,0 \%$ & $68,46 \%$ & $76,66 \%$ \\
\hline Maipú & $48,2 \%$ & $42,96 \%$ & $49,14 \%$ \\
\hline La Florida & $44,4 \%$ & $42,15 \%$ & $48,58 \%$ \\
\hline La Cisterna & $39,5 \%$ & $48,44 \%$ & $54,19 \%$ \\
\hline La Reina & $32,6 \%$ & $52,12 \%$ & $57,73 \%$ \\
\hline San Miguel & $30,0 \%$ & $45,39 \%$ & $48,63 \%$ \\
\hline Santiago & $24,0 \%$ & $47,99 \%$ & $52,80 \%$ \\
\hline N̄uńoa & $12,6 \%$ & $47,72 \%$ & $52,69 \%$ \\
\hline Providencia & $12,1 \%$ & $60,90 \%$ & $65,29 \%$ \\
\hline Las Condes & $8,4 \%$ & $70,08 \%$ & $75,86 \%$ \\
\hline Vitacura & $3,6 \%$ & $76,15 \%$ & $81,30 \%$ \\
\hline
\end{tabular}

Fuente: Elaboración propia con datos encuesta CASEN 2006 y resultados electorales oficiales de la segunda vuelta 2005 y 2009, sobre votos válidamente emitidos.

En el caso de las clases medias, si se analizan los resultados por comunas de la segunda vuelta del 2005 y 2009 . Las comunas que muestran aumentos de un $6 \%$ en el voto de derecha con comunas principalmente de composición mixta, en la cual el porcentaje de clase baja y clase media baja supera el 70\%, mientras que en las comunas con mayor porcentaje de clase media clásica muestran cifras más moderadas. 
Cuadro 2. Comunas por porcentaje que jefes de hogar clasificados como clase media baja - clase media clásica y votación de derecha.

\begin{tabular}{|c|c|c|c|c|}
\hline Comunas & Clase Media Baja & $\begin{array}{c}\text { Clase Media } \\
\text { Clásica }\end{array}$ & Derecha 2005 & Derecha 2009 \\
\hline Pirque & $11,4 \%$ & $11,2 \%$ & $57,03 \%$ & $60,19 \%$ \\
\hline Padre Hurtado & $18,3 \%$ & $4,2 \%$ & $40,46 \%$ & $45,20 \%$ \\
\hline La Pintana & $21,0 \%$ & $1,8 \%$ & $36,50 \%$ & $41,57 \%$ \\
\hline $\begin{array}{l}\text { San José De } \\
\text { Maipo }\end{array}$ & $12,0 \%$ & $11,0 \%$ & $45,21 \%$ & $51,46 \%$ \\
\hline San Ramón & $19,4 \%$ & $5,0 \%$ & $39,47 \%$ & $44,01 \%$ \\
\hline Cerro Navia & $21,0 \%$ & $3,7 \%$ & $37,39 \%$ & $43,80 \%$ \\
\hline La Granja & $19,3 \%$ & $6,8 \%$ & $39,30 \%$ & $44,33 \%$ \\
\hline San Bernardo & $18,0 \%$ & $8,9 \%$ & $44,69 \%$ & $50,66 \%$ \\
\hline Huechuraba & $16,8 \%$ & $11,4 \%$ & $39,13 \%$ & $47,06 \%$ \\
\hline Lo Prado & $20,8 \%$ & $8,5 \%$ & $39,53 \%$ & $45,41 \%$ \\
\hline Renca & $22,0 \%$ & $7,4 \%$ & $39,45 \%$ & $45,47 \%$ \\
\hline El Bosque & $23,7 \%$ & $7,8 \%$ & $43,95 \%$ & $49,62 \%$ \\
\hline Peñalolén & $16,3 \%$ & $15,3 \%$ & $40,25 \%$ & $47,11 \%$ \\
\hline Cerrillos & $18,3 \%$ & $13,7 \%$ & $47,76 \%$ & $50,96 \%$ \\
\hline Lo Espejo & $23,5 \%$ & $10,7 \%$ & $37,42 \%$ & $40,98 \%$ \\
\hline Quinta Normal & $22,4 \%$ & $12,2 \%$ & $42,68 \%$ & $48,40 \%$ \\
\hline Recoleta & $22,8 \%$ & $12,3 \%$ & $43,49 \%$ & $49,36 \%$ \\
\hline Estación Central & $23,2 \%$ & $13,2 \%$ & $41,84 \%$ & $47,00 \%$ \\
\hline $\begin{array}{ll}\text { Pedro } & \text { Aguirre } \\
\text { Cerda } & \\
\end{array}$ & $24,1 \%$ & $13,1 \%$ & $36,53 \%$ & $40,37 \%$ \\
\hline Conchalí & $24,9 \%$ & $13,1 \%$ & $42,18 \%$ & $48,11 \%$ \\
\hline Pudahuel & $26,3 \%$ & $11,7 \%$ & $39,09 \%$ & $45,56 \%$ \\
\hline Puente Alto & $18,9 \%$ & $19,3 \%$ & $42,26 \%$ & $48,80 \%$ \\
\hline Quilicura & $28,9 \%$ & $10,2 \%$ & $41,47 \%$ & $48,81 \%$ \\
\hline Lo Barnechea & $15,7 \%$ & $24,3 \%$ & $68,46 \%$ & $76,66 \%$ \\
\hline San Joaquín & $31,9 \%$ & $10,0 \%$ & $38,81 \%$ & $43,63 \%$ \\
\hline Independencia & $36,3 \%$ & $8,3 \%$ & $46,63 \%$ & $52,86 \%$ \\
\hline Macul & $25,4 \%$ & $19,7 \%$ & $42,82 \%$ & $48,56 \%$ \\
\hline Maipú & $25,0 \%$ & $20,8 \%$ & $42,96 \%$ & $49,14 \%$ \\
\hline La Florida & $21,5 \%$ & $25,9 \%$ & $42,15 \%$ & $48,58 \%$ \\
\hline La Cisterna & $25,4 \%$ & $27,5 \%$ & $48,44 \%$ & $54,19 \%$ \\
\hline La Reina & $12,4 \%$ & $42,5 \%$ & $52,12 \%$ & $57,73 \%$ \\
\hline San Miguel & $23,2 \%$ & $33,2 \%$ & $45,39 \%$ & $48,63 \%$ \\
\hline Vitacura & $3,9 \%$ & $57,9 \%$ & $76,15 \%$ & $81,30 \%$ \\
\hline Las Condes & $5,7 \%$ & $64,6 \%$ & $70,08 \%$ & $75,86 \%$ \\
\hline Santiago & $23,4 \%$ & $47,6 \%$ & $47,99 \%$ & $52,80 \%$ \\
\hline Providencia & $19,6 \%$ & $53,9 \%$ & $60,90 \%$ & $65,29 \%$ \\
\hline Ñuñoa & $21,3 \%$ & $57,7 \%$ & $47,72 \%$ & $52,69 \%$ \\
\hline
\end{tabular}

Fuente: Elaboración propia con datos encuesta CASEN 2006 y resultados electorales oficiales de la segunda vuelta 2005 y 2009, sobre votos válidamente emitidos.

En ese sentido, los resultados permiten afirmar que la composición de clase de las comunas tiene un impacto en el apoyo electoral de la derecha, aunque se observa una disminución de la fuerza de esta relación hacia la última elección. Estos cambios, si bien moderados, deben ser comprendidos partir de una mirada que busque dar luz sobre cómo se configurará el mapa político chileno en los próximos años, atendiendo al comportamiento electoral como un indicador de transformaciones que sobrepasan la 
coyuntura y que marcan tendencias a largo plazo en la forma en que los chilenos pensarán y actuarán políticamente. Para entender este cambio, es preciso avanzar más allá de los números: hay escuchar a los sujetos hablar.

\section{IDENTIFICACIÓN Y ACCIÓN POLÍTICA. CLASES MEDIAS Y TRABAJADORES HOY}

Pensar y actuar políticamente está estrechamente relacionado con la forma en que los sujetos se posicionan en una determinada trama de relaciones, por lo que un análisis de este fenómeno tiene que comenzar por establecer en una primera instancia de esos procesos de diferenciación que estructuran una posición a partir del cual el sujeto comprende y se sitúa en el campo político, así como las articulaciones identitarias que se encuentran tras ésta. En ese marco, los cambios observados en la forma de actuar políticamente están vinculados a modificaciones sustanciales en la forma de concebir el propio espacio en la sociedad: no resulta extraño entonces que los sectores que más han modificado su posición en las últimas décadas en términos de estratificación social sean quiénes muestran mayores cambios a este respecto.

\subsection{De proletariado a clase media. Articulaciones identitarias en la clase baja}

En términos políticos, estos grupos habían estado históricamente ligados a la izquierda a través de una interpelación articulada en torno a las ideas de proletariado, trabajadores y pueblo. Orgánicamente, se vincularon a los partidos a través de su participación en actividades sindicales y durante el período dictatorial, a través de iniciativas como las organizaciones de sobrevivencia económica, organizaciones sindicales y la iglesia.

Durante dicho período, la profunda crisis de desempleo empujó a muchos de ellos a modalidades precarizadas e informales de empleo, en sectores económicos en los que antes participaban: La idea de trabajadores fue medianamente reemplazada en los discursos políticos de la izquierda por la idea de excluidos, modificando sustantivamente a relación entre estos sectores y los partidos políticos (Castillo, 2009).

Con los partidos políticos en clandestinidad y las altas tasas de desempleo, las organizaciones de carácter territorial se convirtieron en el principal espacio de socialización y acción política. La importancia de lo territorial en la construcción de posiciones políticas y en la forma de concebir la propia identidad se visibilizó a partir de la importancia discursiva de figura del poblador en las interpelaciones políticas de la oposición a la dictadura. Estas interpelaciones fueron construyendo el espacio local como lugar de intervención política y al poblador como el actor primordial de éste.

Es precisamente este desplazamiento de la acción política a lo local el marco en el que la estrategia política de la derecha gremial comenzó a mostrar sus primeros frutos. Durante el período dictatorial se realizan los primeros acercamientos entre la derecha y los sectores populares, a través de la primera estrategia sistemática de inserción dirigida a esta población desarrollada por un partido de derecha (Soto, 2001). Tal iniciativa, liderada por la Unión Demócrata Independiente (UDI), marcó los primeros pasos a una estrategia que daría sus frutos a partir de la década de los noventa y que se centraría precisamente en 
una inserción de carácter local en poblaciones marginales. La acogida y lenta penetración de la derecha en estos sectores (Arriagada, 2005; Correa, 2004; Huneeus, 2001; Joignant y Navia, 2003; Castillo, 2009), marcó un giro en la relación histórica entre la clase baja y partidos de izquierda y evidenció transformaciones importantes: el desplazamiento del espacio político al espacio local, el declive de la interpelación de clase y el cambio de la forma en que estos sectores estaban pensando su lugar en la sociedad.

Los cambios observados en la posición y acción política de estos grupos a partir del período de transición muestran modificaciones en la autoidentificación de los individuos y la determinación de aliados y adversarios políticos. Esto se manifiesta en el análisis de las entrevistas para este segmento, donde es posible observar varios elementos interesantes en la construcción de identidades:

1. La autodefinición en los sujetos espacio social está marcada por el componente laboral, pero se observan cambios relevantes en el rol que este elemento desempeña. Disminuye la identificación con el espacio laboral específico, articulándose en torno a la asociación entre trabajo y esfuerzo, dignidad y el "ser responsable".

2. En relación a la forma de concebir el propio lugar en la sociedad, existen diferencias a partir del tipo de ocupación. Quienes poseen más de 50 ańos y que se mantienen en posiciones calificadas del sector primario mantienen una narrativa respecto a la idea de ser "los que están abajo" y "los abusados". Bajo ese rango de edad y en ocupaciones de carácter más inestable, tienen un discurso más basado en la idea de "saber ganarse el pan", asociado al hecho de venir desde posiciones más bajas, conocer la pobreza y haber logrado salir de ella "siendo un luchador".

3. En los entrevistados de empleo más precario, la autodefinición está marcada por la pertenencia a una clase media, un lugar construido a partir de atributos como: "tengo mis cositas", "no me falta nada", "me cuesta ganarme la plata". Acá es posible ver la penetración del discurso meritocrático propio de la narrativa sobre la clase media: en estos entrevistados existe un fuerte énfasis en la movilidad social vía el esfuerzo individual, poniéndose ellos mismos como ejemplo de lo mismo y poniendo como eje "que se puede mejorar con trabajo".

4. En términos de socialización y acción política, es notorio si bien una parte de los entrevistados participa en organizaciones sociales y políticas (tres de un total de ocho), la relación primordial con el sistema político se da a través de la asistencia municipal, existiendo una fuerte crítica a aquellos servicios asociados al estado centralizado como la salud y la educación. Los entrevistados que participan políticamente comparten una percepción asociada a la instrumentalización de las personas y organizaciones por parte de los partidos políticos, ya sean de izquierda o de derecha.

5. Todos los entrevistados comparten una narrativa articulada en torno a la idea "impotencia" o "incapacidad de influir en la sociedad". Comparten la idea de que antes (Período de la Unidad Popular - Dictadura) existía un mayor interés en hacerlos partícipes de los procesos políticos. Quienes participan en organizaciones sociales y políticas asumen 
una postura pragmática frente a esto, señalando que la única forma de conseguir ayuda es establecer relaciones con todos los sectores políticos que la ofrezcan.

\section{2. ¿Radicales, masones y bomberos? Articulaciones identitarias en la clase media}

Las clases medias en Chile se constituyeron mayoritariamente a partir del aumento del aparato burocrático estatal, el que fue su principal empleador hasta 1973, siendo también relevante el rol de la pequeńa industria y comercio, así como el de la inmigración. Entre 1920 y 1973, la literatura les atribuye un rol central en la dinámica del sistema político, pese a su tamaño reducido, a partir de su vínculo estrecho con partidos de centro e izquierda (Faletto, 1980; Lomnitz, 1998). Dentro de esta línea, se ha señalado que las clases medias se habrían constituido en una pieza clave en la conformación de alianzas pluripartidistas, como es el caso del Frente Popular de 1938, a partir de su participación en partidos como el Partido Radical (PR), la Democracia Cristiana (DC) y parte de las bases de apoyo del Partido Socialista. La relativa unidad política de estos sectores - pese a su heterogeneidad- estuvo dada por su estrecho vínculo con el estado y con una red organizacional asociada al ejercicio de determinadas profesiones, constituyéndose éstos en sus principales espacios políticos, a través de una intensa actividad en organizaciones de funcionarios públicos (Candina, 2013) o en la administración de los gobiernos radicales y demócrata-cristianos. A partir de las reformas estructurales esta situación comenzó a transformarse. Uno de los primeros efectos sobre las clases medias se dio a partir de la drástica disminución del aparato estatal, en el marco de la contracción del gasto público: una buena parte de la población antes vinculada laboralmente al estado transitó hacia la empresa privada, siendo ésta el principal empleador de las clases medias hoy. Sumado a esto, se observa un importante aumento del peso relativo de este sector en la estructura ocupacional del país. Este sector pasó del 18 por ciento de la PEA en 1971, a un 22 por ciento en 1987 y a un 27 por ciento en 1995 (León y Martínez, 2001: 15).

En términos políticos, las clases medias se vincularon durante la dictadura mayoritariamente a la oposición, a pesar de que uno de los partidos apuntados como con una mayor composición de clase media, el Partido Demócrata Cristiano, manifestó públicamente su apoyo al régimen en una primera instancia. A partir de la crisis económica, segmentos dentro de las clases medias participaron en las Jornadas de Protesta de 1983-1984, relacionándose orgánicamente con los grupos más moderados de la oposición: los partidos de izquierda en proceso de renovación ideológica (fracciones del Partido Socialista, del partido Comunista y del Movimiento de Acción Popular Unitaria) y con la Democracia Cristiana. Hacia finales del período dictatorial, estas organizaciones políticas conformaron el bloque Concertación de Partidos por la Democracia. Disgregada en el sector privado bajo diversas y nuevas formas de empleo, la organización sindical, gremial o política de las clases medias se volvió sumamente dificultosa dada la multiplicidad de condiciones laborales, ingresos, intereses y estilos de vida. La unidad política en la heterogeneidad, dada antes por la condición de empleados estatales o por la condición de oposición al régimen dictatorial, comenzó a tambalear. Esto también se vio acrecentado a partir de la agudización de la brecha en el seno mismo de las clases medias, marcada por la una diferencia sustantiva entre la ocupación, ingreso y status entre las clases medias consolidadas y las clases medias en proceso de consolidación. Uno de los ejes diferenciadores centrales, como se observa a continuación en los resultados del análisis cualitativo, tiene que ver con las trayectorias de movilidad social. Dentro de estos hallazgos, destaca: 
1. Existen notorias diferencias en los atributos que los entrevistados de las dos clases medias (clásica y baja) destacan en la definición de sí mismos. En el caso de la clase media clásica, existe una tendencia a centrarse en la profesión, destacando generalmente las trayectorias de movilidad social a través de la educación y el "origen humilde", la austeridad y simpleza. En el caso de la clase media baja, la autodefinición está asociada a idea de "persona con ambición", que "quiere surgir", poniendo especial énfasis en lo "sacrificado" del proceso de movilidad social.

2. Entre quienes pertenecen a la clase media clásica, interpelados por quiénes se encuentran en posiciones distintas a la suya, la primera identificación de un "otro" significativo se encuentra asociada a quienes tienen más dinero: "los jefes", "los dueños", connotados negativamente como "anticuado", "rancio" o que "viven de las apariencias". Posteriormente identifican a quienes se encuentran en posiciones más bajas, connotados positivamente por atributos como "gente muy esforzada", "trabajadora", "gente muy modesta" en condiciones "complicadas" y "entornos difíciles". En el caso de las clases medias bajas, las posiciones altas son connotadas con atributos positivos, como personas "que saben qué hacer con su plata", "los que la llevan", mientras que los de posiciones más bajas están mucho más asociados a atributos negativos: son "gente que tiene cero ambición y se dedican a delinquir", "conformistas", "que viven en las drogas".

3. En términos de participación política, en el caso de la clase media clásica, si bien hay un rescate "discursivo" de diversas formas de participación social, pero sólo uno de los entrevistados pertenece a alguna participación política. La relación con los organismos del sistema político se reduce al pago de impuestos y trámites burocráticos, pero en general es un segmento relativamente autónomo de estos servicios, por lo que manifiesta una postura desinteresada en torno a la política, articulada en torno a las ideas de "está mal pero no me afecta", "no tengo las herramientas para cambiarlo y no veo para qué". En el caso de la clase media baja, más que un desinterés hay un cierto rechazo a la práctica de lo político, asociada a la identificación de una acción del estado que perjudica la clase media, "obedece a los ricos" y "le da plata a los pobres" pero no otorga espacio ni ayuda a "los que estamos al medio".

\section{CONCLUSIONES}

Los datos presentados plantean una vía para comprender el ascenso de la derecha a partir de dos puntos: el primero de ellos, que el apoyo electoral a esta coalición habla de transformaciones relevantes en la forma en que los chilenos están pensando políticamente. Esto se observa una lenta reestructuración del patrón histórico de relación entre clases y partidos que establecía una relación de oposición entre clase baja-clases medias y las organizaciones políticas de derecha. Esto plantea importante preguntas a futuro sobre cómo hacer política en Chile y cuál será la relación de la ciudadanía con el sistema político en general y con los partidos políticos en particular, preguntas que son particularmente relevantes en el marco de la oleada de protestas que ha vivido Chile durante la primera mitad del año 2011. 
El segundo punto que se ha tratado de establecer es que esta transformación en la forma de pensar políticamente y de relacionarse con los partidos, está vinculada con aquellos cambios en la estratificación social que modificaron de manera sustantiva no sólo porque alteró la configuración misma de las clases medias y la clase baja, sino también su experiencia cotidiana y su percepción de lugar en la sociedad. Así por ejemplo, en la clase baja el análisis cualitativo habla claramente de una percepción de exclusión e impotencia que hace a estos grupos particularmente susceptibles establecer relaciones clientelares con los partidos con única forma de conseguir ayuda. Esta percepción contrasta con la idea de que antes "eran escuchados" o por lo menos se les intentaba controlar o intervenir. La sensación de abandono -caldo de cultivo para la penetración de un discurso anclado en el clientelismo- muestra claramente cuál es el lugar que la clase baja percibe como suyo en la sociedad actual y en el sistema político.

De la misma manera, en las clases medias las narrativas nos hablan por un lado de una clase media clásica privatizada, que se ha independizado del estado y del sistema político y una clase media baja que -muy a su pesar-también debe asegurar su status de manera individual. Esto redunda en una penetración importante de la narrativa anclada en la movilidad social rápida, en la figura del "emprendedor" y del "esfuerzo" que sin duda se ha reforzado, en forma de círculo, a partir de la narrativa de los partidos de derechas que han puesto como eje central estos puntos. En el marco de una clase media privatizada, el ejercicio de la política pierde sentido y las posibilidades de mejorar la calidad de vida propia o de la familia pasa por la acción de cada individuo, razón por la que el apoyo electoral se vuelve volátil y la figura de "los que saben qué hacer con su plata" un modelo poderoso. Es notorio que para segmentos dentro de las clases medias la figura de un empresario exitoso como presidente -Sebastián Piñera- resultaba atractiva por encarnar precisamente parte sustancial de este imaginario de "ganador".

Es claro que sobre este tema queda mucha investigación por realizar, tanto en el terreno de las nuevas articulaciones identitarias como en el campo de cómo esto impacta en el comportamiento político y electoral de estos grupos. Iniciar este camino es hoy más urgente que nunca, en el contexto de una sociedad que comienza a rearticularse y configurar un nuevo mapa de actores políticos. La tarea es grande. Todo aporte es bienvenido.

\section{REFERENCIAS}

Altman, David. "Redibujando el mapa electoral chileno: Incidencia de factores socioeconómicos y género en las urnas", Revista de Ciencia Politica XXIV/2 (2004): 49-66.

Aninat, Cristóbal y Elacqua, Gregory. El electorado que definió las elecciones. Santiago de Chile: Expansiva, 2010.

Arriagada, Evelyn. "UDI: ¿Partido popular o partido populista? Consideraciones sobre el éxito electoral de Partido Unión Demócrata Independiente en los sectores populares", Colección IDEAS 51 (2005). 
Atria, Raúl. "Estructura ocupacional, estructura social y clases sociales", Serie de Políticas Sociales 96. Santiago de Chile. CEPAL. División de Desarrollo Social, 2004.

Barozet, Emmanuelle."El valor histórico del pituto: Clase media, integración y diferenciación social en Chile”, Revista de Sociología 20 (2006): 69-96.

Barozet, Emmanuelle y Espinoza, Vicente. “¿De qué hablamos cuando decimos "clase media”? Perspectivas sobre el caso chileno", El Arte de Clasificar a los Chilenos, Expansiva-UDP-La Tercera, (2009), Santiago de Chile.

Barozet, Emmanuelle y Fierro, Jaime. "Clase media en Chile, 1990-2011: algunas implicancias sociales y políticas”, Serie Estudios 4 (2011), Konrad Adenauer Stiftung, Santiago de Chile.

Candina, Azún. Clase media, Estado y sacrificio. Santiago de Chile: LOM ediciones, 2013.

Castillo, Mayarí. Identidades políticas. Trayectorias y cambios en el caso chileno. Ciudad de México: Facultad Latinoamericana de Ciencias Sociales, 2009.

"El centro de la disputa: clases medias y la política de la desigualdad en Chile". En Mayarí Castillo, Anahí Durand y Manuel Bastias (Comps.), Desigualdad, Legitimación y conflicto. Dimensiones politicas y culturales de la desigualdad en América Latina. Santiago de Chile: Ediciones Universidad Alberto Hurtado, 2011.

Correa, Sofía. Con las riendas del poder. La derecha chilena en el siglo XX. Santiago de Chile: Editorial Sudamericana, 2004.

Cristi, Pablo. “¿Dónde están los alcaldes UDI electos en el 2000? Desempeño electoral de la UDI 2000-2004”, Documento de trabajo, 25. Observatorio Electoral. ICSO Universidad Diego Portales, 2008.

Crompton, Mary. Clase y estratificación. Madrid: Tecnos, 1997.

Dávila, Mireya y Fuentes, Claudio. Promesas de cambio: Izquierda y derecha en el Chile Contemporáneo. Santiago de Chile: Flacso-Chile, 2002.

Espinoza, Vicente. "La movilidad ocupacional en el Cono sur. Oportunidades y desigualdad social”, Revista de Sociología 20 (2006): 131-146.

Espinoza, Vicente; Barozet, Emmanuelle y Méndez, María Luisa. "Estratificación y movilidad social bajo un modelo neoliberal: El caso de Chile", Revista Lavboratorio, 25/14 (2013): 169-191.

Faletto, Enzo. "Algunas características de la base social del partido socialista y del partido comunista 1958-1973", Documento de Trabajo 97. Santiago de Chile: FlacsoChile, 1980. 
Faletto, Enzo. "Clases sociales y opciones políticas en Chile", Documentos de trabajo 130. Santiago de Chile: FLacso-Chile, 1982.

Fediakova, Eugenia. "Conservadores e innovadores: La derecha en la segunda mitad del siglo XX”. En Mireya Dávila y Claudio Fuentes (Eds.), Promesas de cambio: Izquierda y derecha en el Chile Contemporáneo. Santiago de Chile: Flacso-Chile, 2002.

Glaser, Barney G. 1998. Doing grounded theory: Issues and discussions. Mill Valley, CA: Sociology Press.

Huneeus, Carlos. "La derecha en Chile después de Pinochet: El caso de la Unión Demócrata Independiente", Documento de trabajo, 285. París, Kellog Institute - Universidad de Notre Dame, 2001.

Joignant, Jorge y Navia, Patricio. "De la política de individuos a los hombres del partido: socialización, competencia política y penetración electoral de la UDI (19892001)”, Revista Estudios Públicos 89 (2003).

Johnson, John. "Atrincheramiento político de los sectores medios en Chile". En Hernán Godoy, Estructura Social de Chile. Santiago de Chile: Editorial Universitaria, 1971.

León, Arturo y Martínez, Javier. "La estratificación social chilena hacia fines del siglo XX", Documento de trabajo, Serie Políticas Sociales 52 (2001): 3-41. Santiago de Chile: CEPAL/ECLAC.

Lomnitz, Larissa y Melnick, Ana. La cultura política chilena y los partidos de centro. Fondo de Cultura Económica México. Santiago de Chile: Fondo de Cultura Económica México, 1998.

Mainwaring, Scott y Torcal, Mariano. "El conflicto democracia/autoritarismo y sus bases sociales en Chile, 1973-1995: Un ejemplo de redefinición política de un cleavage”, Reis 103 (2003): 51-82.

Martínez, Javier y Tironi, Eugenio. Las clases sociales en Chile. Cambio y estratificación, 19701980. Colección Estudios Sociales. Santiago de Chile: Ediciones SUR, 1985.

Méndez, María Luisa y Gayo, Modesto. "El perfil de un debate: movilidad y meritocracia. Contribución al estudio de las sociedades latinoamericanas". En rolando Franco, Arturo León y Raúl Atria (Coords.), Estratificación y movilidad social en América Latina. Santiago de Chile: LOM Ediciones, (2007): 121-157.

Minujin, Alberto y Comas, Guillermina."Vulnerability and Resilience of the Middle Class in Latin America”, International Affairs Working Paper. The New School, 2009. 
Morales, Mauricio. "Zorros" y "Leones" en la Derecha Política Chilena. La coalición de partidos UDI-RN, 1989-2001. Tesis de maestro en Ciencias Sociales. México: Flacso- México, 2004.

Morales, Mauricio y Cantillana, Carlos.“ ¿Me verás caer? La UDI frente a las municipales del 2008”, Documento de trabajo 19. Observatorio Electoral. Santiago de Chile: ICSO - Universidad Diego Portales, 2008.

Moulian, Tomás y Torres, Isabel. 1989. "La problemática de la derecha política en Chile, 1973-1983”. En Marcelo Cavarozzi y Manuel Antonio Garretón (Coords.), Muerte y resurrección. Los partidos politicos en el autoritarismo y las transiciones en el cono sur. Santiago de Chile: Flacso-Chile, (1989): 335- 393.

Portes, Alejandro y Hoffman, Nelly. "Las estructuras de clase en América latina: composición y cambios durante la época neoliberal", Serie políticas sociales 68. Santiago de Chile: CEPAL, 2003.

Rojas, Priscilla. "Historia de la Alianza por Chile. Elecciones Municipales 1992 - 2004", Documento de trabajo, 5. Santiago de Chile: Observatorio Electoral. ICSO Universidad Diego Portales, 2008.

Sémbler, Camilo. "Estratificación social y clases sociales. Una revisión analítica de los sectores medios", Serie Políticas Sociales 25. Santiago de Chile: División de Desarrollo Social - CEPAL, 2006.

Sisto, Vicente y Fardella, Carla. "Control narrativo y gubernamentalidad: la producción de coherencia en las narrativas identitarias. El caso de profesionales chilenos adultos jóvenes en condiciones de vinculación laboral flexible", FORUM. Qualitative Social Research 10/2 (2009) artículo 29.

Scully, Timothy. Rethinking the Center: Cleavages, Critical Junctures, and Party Evolution in Chile. California: Stanford University Press, 1992.

Silva, Beatriz. "La clase media después de las transformaciones estructurales: una aproximación cualitativa a través del análisis de clase". Tesis para obtener el grado de socióloga. Santiago de Chile: Universidad de Chile, 2005.

Soto, Ángel. “La irrupción de la UDI en las poblaciones 1983-1987”. Ponencia presentada a sesión de Latin American Studies Association. Washington DC, 2001.

Tironi, Eugenia; Agüero, Felipe y Valenzuela, Eduardo. 2001. "Clivajes políticos en Chile: Perfil sociológico de los electores de Lagos y Lavín”, Revista Perspectivas 5/1 (2001): 73-87. 
Torcal, Mariano y Mainwaring, Scott. "The Political Recrafting of Social Bases of Party Competition: Chile, 1973-95”, British Journal of Political Science 33/1 (2003): 55-84. Cambridge. Cambridge University Press.

Torche, Florencia. Desigual pero fluido: El patrón chileno de movilidad en perspectiva comparada. Santiago de Chile: Serie en Foco, Investigaciones Expansiva, 2005.

Torche, Florencia y Wormald, Guillermo. "Chile, entre la adscripción y el logro", en Estratificación y Movilidad Social en América Latina. Transformaciones estructurales de un cuarto de siglo. Santiago de Chile: LOM Ediciones, 2007.

Valenzuela, Samuel. "Orígenes y transformaciones del sistema de partidos en Chile", Revista Estudios Públicos 58. Santiago de Chile: Centro de Estudios Públicos, 1995.

Valenzuela, Arturo. "Orígenes y características del sistema de partidos en Chile: proposición para un gobierno parlamentario", Estudios Públicos 64, Santiago de Chile: Centro de Estudios Públicos, 1996.

Winn, Peter (Ed.), Victims of the Chilean Miracle: Workers and Neoliberalism in the Pinochet Era. 1973 - 2002. Durham, NC: Duke University Press, 2004.

Wright, O. Erik. Classes. London: Verso, 1985.

-----------. "Understanding Class. Towards an Integrated Analyttical Approach", New Left Review 60 (2009): 101-116.

Zeitlin, Maurice y James Petras. "The Working-Class Vote in Chile: Christian Democracy versus Marxism”, British Journal of Sociology 21 (1970): 16-29. 